\title{
X-RAY EMISSION FROM SUPERNOVAE IN DENSE CIRCUMSTELLAR MATTER ENVIRONMENTS: A SEARCH FOR COLLISIONLESS SHOCKS
}

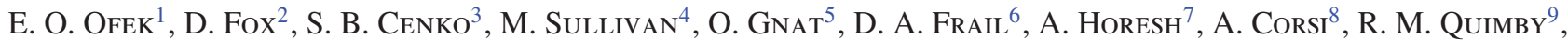

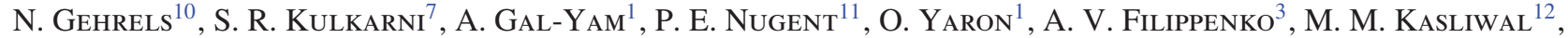 \\ L. Bildsten ${ }^{13,14}$, J. S. Bloom ${ }^{3}$, D. Poznanski ${ }^{15}$, I. ArCavi ${ }^{1}$, R. R. Laher ${ }^{16}$, D. Levitan ${ }^{7}$, B. Sesar ${ }^{7}$, And J. Surace ${ }^{16}$ \\ ${ }^{1}$ Benoziyo Center for Astrophysics, Weizmann Institute of Science, 76100 Rehovot, Israel \\ ${ }^{2}$ Department of Astronomy and Astrophysics, Pennsylvania State University, University Park, PA 16802, USA \\ ${ }^{3}$ Department of Astronomy, University of California, Berkeley, CA 94720-3411, USA \\ ${ }^{4}$ Department of Physics, University of Oxford, Denys Wilkinson Building, Keble Road, Oxford OX1 3RH, UK \\ ${ }^{5}$ Racah Institute of Physics, The Hebrew University of Jerusalem, 91904 Jerusalem, Israel \\ ${ }^{6}$ National Radio Astronomy Observatory, P.O. Box O, Socorro, NM 87801, USA \\ ${ }^{7}$ Division of Physics, Mathematics, and Astronomy, California Institute of Technology, Pasadena, CA 91125, USA \\ ${ }^{8}$ LIGO Laboratory, Division of Physics, California Institute of Technology, MS 100-36, Pasadena, CA 91125, USA \\ ${ }^{9}$ Kavli IPMU, University of Tokyo, 5-1-5 Kashiwanoha, Kashiwa-shi, Chiba 277-8583, Japan \\ 10 NASA-Goddard Space Flight Center, Greenbelt, MD 20771, USA \\ ${ }^{11}$ Lawrence Berkeley National Laboratory, 1 Cyclotron Road, Berkeley, CA 94720, USA \\ 12 Observatories of the Carnegie Institution for Science, 813 Santa Barbara Street, Pasadena, CA 91101, USA \\ ${ }^{13}$ Kavli Institute for Theoretical Physics, Kohn Hall, University of California, Santa Barbara, CA 93106, USA \\ ${ }^{14}$ Department of Physics, Broida Hall, University of California, Santa Barbara, CA 93106, USA \\ ${ }^{15}$ School of Physics and Astronomy, Tel Aviv University, Tel Aviv, Israel \\ ${ }^{16}$ Spitzer Science Center, California Institute of Technology, MS 314-6, Pasadena, CA 91125, USA \\ Received 2012 May 30; accepted 2012 October 18; published 2013 January 4
}

\begin{abstract}
The optical light curve of some supernovae ( $\mathrm{SNe}$ ) may be powered by the outward diffusion of the energy deposited by the explosion shock (the so-called shock breakout) in optically thick $(\tau \gtrsim 30)$ circumstellar matter (CSM). Recently, it was shown that the radiation-mediated and radiation-dominated shock in an optically thick wind must transform into a collisionless shock and can produce hard X-rays. The X-rays are expected to peak at late times, relative to maximum visible light. Here we report on a search, using Swift/XRT and Chandra, for X-ray emission from $28 \mathrm{SNe}$ that belong to classes whose progenitors are suspected to be embedded in dense CSM. Our sample includes 19 Type IIn SNe, one Type Ibn SN, and eight hydrogen-poor superluminous SNe (SLSN-I such as SN 2005ap). Two SNe (SN 2006jc and SN 2010jl) have X-ray properties that are roughly consistent with the expectation for X-rays from a collisionless shock in optically thick CSM. However, the X-ray emission from SN 2006jc can also be explained as originating in an optically thin region. Thus, we propose that the optical light curve of SN 2010j1 is powered by shock breakout in CSM. We suggest that two other events (SN 2010al and SN 2011ht) were too X-ray bright during the SN maximum optical light to be explained by the shock-breakout model. We conclude that the light curves of some, but not all, SNe IIn/Ibn are powered by shock breakout in CSM. For the rest of the SNe in our sample, including all of the SLSN-I events, our X-ray limits are not deep enough and were typically obtained too early (i.e., near the SN maximum light) for definitive conclusions about their nature. Late-time X-ray observations are required in order to further test whether these $\mathrm{SNe}$ are indeed embedded in dense CSM. We review the conditions required for a shock breakout in a wind profile. We argue that the timescale, relative to maximum light, for the $\mathrm{SN}$ to peak in X-rays is a probe of the column density and the density profile above the shock region. In SNe whose X-ray emission slowly rises, and peaks at late times, the optical light curve is likely powered by the diffusion of shock energy in a dense CSM. We note that if the CSM density profile falls faster than a constant-rate wind-density profile, then X-rays may escape at earlier times than estimated for the wind-profile case. Furthermore, if the CSM has a region in which the density profile is very steep relative to a steady wind-density profile, or if the CSM is neutral, then the radio free-free absorption may be sufficiently low for radio emission to be detected.
\end{abstract}

Key words: stars: mass-loss - supernovae: general - supernovae: individual (SN 2006jc, SN 2010jl)

Online-only material: machine-readable table

\section{INTRODUCTION}

Circumstellar matter (CSM) around supernova (SN) progenitors may play an important role in the emission and propagation of energy from SN explosions. The interaction of the SN radiation with optically thin CSM shells may generate emission lines, with widths that are representative of the shell velocity or the velocity of the shocked cool gas in the post-shock region, as in Type IIn SNe (SNe IIn; Schlegel 1990; Kiewe et al. 2012; see Filippenko 1997 for a review of SN classification). The interac- tion of SN ejecta with the CSM can power the light curves of SNe by transformation of the SN kinetic energy into photons. In cases where a considerable amount of optically thin (and ionized) material is present around the exploding star, synchrotron, and free-free radiation can emerge, and inverseCompton scattering can generate X-ray photons (e.g., Chevalier \& Fransson 1994; Horesh et al. 2012; Krauss et al. 2012).

For the Type IIn SN PTF 09uj, Ofek et al. (2010) suggested that a shock breakout can take place in an optically thick wind (see also Grassberg et al. 1971; Falk \& Arnett 1977; Chevalier 
\& Irwin 2011; Balberg \& Loeb 2011). This will happen if the Thomson optical depth within the wind profile is $\gtrsim c / v_{\mathrm{sh}}$, where $c$ is the speed of light and $v_{\mathrm{sh}}$ is the shock speed. Ofek et al. (2010) showed that shock breakout in wind environments produces optical displays that are brighter and have longer timescales than those from the surfaces of red supergiants (e.g., Colgate 1974; Matzner \& McKee 1999; Nakar \& Sari 2010; Rabinak \& Waxman 2011; Couch et al. 2011). Chevalier \& Irwin (2011) extended this picture. Specifically, they discussed CSM with a wind profile in which the wind has a cutoff at a distance $R_{\mathrm{W}}$. If the optical depth at $R_{\mathrm{W}}$ is $\lesssim c / v_{\mathrm{s}}$, then the SN light curve will have a slow decay (e.g., SN 2006gy; Ofek et al. 2007; Smith et al. 2007). If the optical depth at $R_{\mathrm{w}}$ is $\gtrsim c / v_{\mathrm{s}}$, then it will have a faster decay (e.g., SN 2010gx; Pastorello et al. 2010a; Quimby et al. 2011b). Moriya \& Tominaga (2012) investigated shock breakouts in general wind-density profiles of the form $\rho \propto r^{-w}$. They suggested that, depending on the power-law index $w$, shock breakouts in wind environments can produce bright SNe without narrow emission lines (e.g., SN 2008es; Gezari et al. 2009; Miller et al. 2009).

Recently Katz et al. (2011) and Murase et al. (2011) showed that if the progenitor is surrounded by optically thick CSM, then a collisionless shock is necessarily formed during the shock breakout. Moreover, they argued that the energy emitted from the collisionless shock in the form of high-energy photons and particles is comparable to the shock-breakout energy. Furthermore, this process may generate high-energy ( $\gtrsim 1 \mathrm{TeV})$ neutrinos. Although Katz et al. (2011) predicted that the photons are generated with energy typically above $60 \mathrm{keV}$, it is reasonable to assume that some photons will be emitted with lower energy due to reprocessing of photons (see below) and the continuum nature of the radiation. Chevalier \& Irwin (2012) showed that Comptonization and inverse-Compton scattering of the highenergy photons is likely to play an important role, and that the high-energy photons will be absorbed.

Svirski et al. (2012) discuss the X-ray emission from collisionless shocks. They show that at early times the X-rays will be processed into the optical regime by the Compton process. Therefore, at early times, the optical emission will be about $10^{4}$ times stronger than the high-energy emission. With time, the $\mathrm{X}$-ray emission will become stronger, while the optical emission will decay. They conclude that for a CSM with a steady wind profile $(w=2)$, X-ray emission may peak only at late times, roughly 10-50 times the shock-breakout timescale. The shock-breakout timescale, $t_{\mathrm{br}}$, is roughly given by the diffusion timescale at the time of shock breakout. This timescale is also equivalent to the radius at which the shock breaks out $\left(r_{\mathrm{br}}\right)$ divided by the shock velocity ( $v_{\mathrm{s}}$; Weaver 1976). If the main source of optical photons is due to diffusion of the shock-breakout energy, the SN optical light rise time, $t_{\text {rise }}$, will be equivalent to the shock-breakout timescale. Therefore, X-ray flux measurements and spectra of SNe embedded in dense CSM starting from the explosion until months or years after maximum light are able to measure the properties of the CSM around the SN progenitors and the progenitor mass-loss history. This unique probe into the final stages of massive star evolution has been only partially exploited, at best.

Herein, we analyze the X-ray data for $28 \mathrm{SNe}$ with light curves that may be powered by a shock breakout from dense CSM, and for which Swift/XRT (Gehrels et al. 2004) observations exist. We use this sample to search for X-ray signatures of collisionless shocks-emission at late times (months to years after peak optical luminosity). We suggest that these signals were observed in several cases, most notably in SN 2010j1 (Chandra et al. 2012b). Finally, we review the conditions for a shock breakout in CSM with a wind profile, and we discuss the importance of bound-free absorption and the possibility of detecting radio emission from such $\mathrm{SNe}$.

The structure of this paper is as follows. In Section 2 we present the SN sample, while Section 3 presents the X-ray observations. We review and discuss the model in Section 4, and consider the observations in the context of the model in Section 5. We summarize our conclusions in Section 6.

\section{SAMPLE}

Our sample is based on SNe found by amateur astronomers and several surveys, including the Lick Observatory Supernova Search (Li et al. 2000; Filippenko et al. 2001), the Catalina Real-time Transient Survey (Drake et al. 2009a), Pan-STARRS1 (PS1; Kaiser et al. 2002), and the Palomar Transient Factory ${ }^{17}$ (PTF; Law et al. 2009; Rau et al. 2009). Two SNe, PTF 09drs and PTF 10tel, are reported here for the first time. We note that many of the nearby or luminous SNe found by PTF are also observed by Swift.

We selected a sample of $\mathrm{SNe}$ in which the main source of energy may be explained by diffusion of the explosion shock energy through optically thick CSM around the progenitor. First, we include SNe IIn within $200 \mathrm{Mpc}$. Objects that belong to this class show relatively narrow (intermediate width) hydrogen emission lines, an indication of the presence of optically thin material somewhere around the progenitor. However, it is unlikely that all SNe showing relatively narrow hydrogen emission lines in their spectra are powered mainly by the diffusion of shock energy in an optically thick environment. One reason is that some $\mathrm{SNe}$ In show X-ray emission near maximum optical light, which is not expected when optically thick CSM is present (see Section 4). Furthermore, Moriya \& Tominaga (2012) suggest that not all SNe powered by interaction of the ejecta with slowly moving material will necessarily have narrow emission lines in their spectrum. We note that some of the SNe IIn in our sample are peculiar (e.g., SN 2010jp/PTF 10aaxi; Smith et al. 2012).

Another relevant, but rare, class of objects are $\mathrm{SNe} \mathrm{Ibn}$. This class is defined by the lack of hydrogen lines and the presence of narrow helium emission lines. The only SN of this type in our sample is SN 2006jc (Nakano et al. 2006; Foley et al. 2007; Pastorello et al. 2008; Smith et al. 2008).

The third class of SNe we investigate here is the small group of hydrogen-poor superluminous SN (SLSN-I; see review in GalYam 2012). Quimby et al. (2011b) used spectra of several such events found by PTF, at intermediate redshift $(z \approx 0.5)$, to show that these events, as well as SCP 06F6 (Barbary et al. 2009) and SN 2005ap (Quimby et al. 2007), are spectroscopically similar. This group of SNe continues to grow with new discoveries (e.g., Chomiuk et al. 2011; Leloudas et al. 2012), and their hosts were studied by Neill et al. (2011). Although the nature of these events is not understood (e.g., Kasen \& Bildsten 2010), Quimby et al. (2011b) suggested that they may be powered by a pulsational pair-instability SN (Rakavy et al. 1967; Woosley et al. 2007). According to this hypothesis, the SN ejecta interact with a dense shell of material, enriched with intermediate-mass elements, that was expelled by the progenitor during previous explosions (see also Ginzburg \& Balberg 2012). This model is tentatively supported by observations of SN 2006oz (Leloudas

\footnotetext{
17 http://www.astro.caltech.edu/ptf/
} 
Table 1

SN Sample

\begin{tabular}{|c|c|c|c|c|c|c|c|c|c|c|c|}
\hline Name & Type & $\begin{array}{r}\alpha_{\mathrm{J} 2000} \\
(\operatorname{deg})\end{array}$ & $\begin{array}{l}\delta_{\mathrm{J} 2000} \\
(\mathrm{deg})\end{array}$ & $\begin{array}{c}t_{\text {rise }} \\
\text { (day) }\end{array}$ & $\begin{array}{c}M_{R} \\
(\mathrm{mag})\end{array}$ & $z$ & $\begin{array}{c}t_{\text {peak }} \\
(\mathrm{MJD})\end{array}$ & $\begin{array}{c}N_{\mathrm{H}} \\
\left(10^{20} \mathrm{~cm}^{-2}\right)\end{array}$ & $\begin{array}{c}L_{\mathrm{X}} \\
\left(\mathrm{erg} \mathrm{s}^{-1}\right)\end{array}$ & $\frac{L_{\mathrm{X}}}{L_{\mathrm{opt}}}$ & $\mathrm{FAP}_{\text {min }}$ \\
\hline PTF 09atu & SLSN-I & 247.60229 & +23.64029 & 30: & -22.5 & 0.501 & 55060 & 4.05 & $<1.9 \times 10^{44}$ & 0.7 & 1.00 \\
\hline PTF 09cnd & SLSN-I & 243.03725 & +51.48782 & 50 & -22.8 & 0.258 & 55080 & 1.67 & $<8.0 \times 10^{42}$ & 0.02 & 1.00 \\
\hline PTF 09cwl/SN 2009jh & SLSN-I & 222.29200 & +29.41983 & 50 & $-22.5:$ & 0.349 & 55060 & 1.51 & $<1.1 \times 10^{44}$ & 0.4 & 1.00 \\
\hline SN 2010gx/PTF 10cwr & SLSN-I & 171.44448 & -8.82810 & 20 & $-21.7:$ & 0.231 & 55280 & 3.78 & $<9.9 \times 10^{42}$ & 0.07 & 1.00 \\
\hline PTF 10hgi & SLSN-I & 249.44601 & +6.20898 & 50 & -20.3 & 0.096 & 55370 & 6.06 & $<5.1 \times 10^{42}$ & 0.1 & 1.00 \\
\hline PTF 11dij & SLSN-I & 207.74069 & +26.27856 & 40: & $-21.1:$ & 0.143 & 55690 & 1.21 & $<4.6 \times 10^{42}$ & 0.06 & 1.00 \\
\hline PTF 11rks & SLSN-I & 24.93962 & +29.92417 & 20 & -21.0 & 0.20 & 55945 & 5.27 & $<5.4 \times 10^{42}$ & 0.07 & 1.00 \\
\hline PS $1-12$ fo & SLSN-I & 146.55379 & +19.84131 & $>14$ & -21.0 & 0.175 & 55956 & 2.79 & $<1.8 \times 10^{43}$ & 0.2 & 1.00 \\
\hline SN 2006jc & Ibn & 139.36667 & +41.90889 & $<15$ & -17.8 & 0.006 & 54020 & 1.00 & $\sim 1.5 \times 10^{41}$ & 0.04 & 0.00 \\
\hline PTF 09drs & IIn & 226.62567 & +60.59427 & 40: & $-17.8:$ & 0.045 & 55210 & 1.72 & $<4.4 \times 10^{42}$ & 1.2 & 1.00 \\
\hline SN 2010j1/PTF 10aaxf & IIn & 145.72221 & +9.49494 & $15 . .23$ & -20.6 & 0.011 & 55500 & 3.05 & $\sim 1.8 \times 10^{41}$ & 0.004 & 0.00 \\
\hline SN 2010jp/PTF 10aaxi & IIn & 94.12770 & -21.41001 & $<19$ & -14.6 & 0.01 & 55520 & 11.0 & $<1.2 \times 10^{40}$ & 0.06 & 0.04 \\
\hline SN 2010jj/PTF 10aazn & IIn & 31.71774 & +44.57156 & $15 . .53$ & -18.0 & 0.016 & 55530 & 9.38 & $<1.2 \times 10^{41}$ & 0.03 & 1.00 \\
\hline SN 2010bq/PTF 10fjh & IIn & 251.73066 & +34.15964 & $15 . .45$ & -18.5 & 0.032 & 55310 & 1.79 & $<1.2 \times 10^{42}$ & 0.2 & 1.00 \\
\hline PTF 11iqb & IIn & 8.52015 & -9.70498 & 10: & -18.4 & 0.013 & 55780 & 2.79 & $\sim 7.9 \times 10^{40}$ & 0.01 & 0.00 \\
\hline SN 2007bb & IIn & 105.28108 & +51.26592 & $<15$ & $-17.6:$ & 0.021 & 54192 & 7.04 & $<2.8 \times 10^{41}$ & 0.09 & 0.07 \\
\hline SN 2007pk & IIn & 22.94613 & +33.61503 & $<14$ & $-17.3:$ & 0.017 & 54423 & 4.72 & $<1 \times 10^{41}$ & 0.04 & 0.00 \\
\hline SN 2008cg & IIn & 238.56313 & +10.97361 & $30 . .60$ & $-19.4:$ & 0.036 & 54583: & 3.65 & $<2.6 \times 10^{41}$ & 0.02 & 1.00 \\
\hline SN 2009au & IIn & 194.94167 & -29.60208 & $\ldots$ & $-16.5:$ & 0.009 & 54901: & 6.42 & $<3.5 \times 10^{40}$ & 0.03 & 0.15 \\
\hline SN 2010al & IIn & 123.56629 & +18.43839 & $<35$ & -16.0 & 0.0075 & 55268: & 3.92 & $\sim 2.2 \times 10^{41}$ & 0.3 & 0.00 \\
\hline SN 2011ht & IIn & 152.04413 & +51.84917 & 50 & -16.8 & 0.004 & 55880 & 0.78 & $\sim 7.2 \times 10^{39}$ & 0.05 & 0.00 \\
\hline SN 2011hw & IIn & 336.56058 & +34.21642 & $\cdots$ & $-19.1:$ & 0.023 & 55883: & 10.2 & $<5.1 \times 10^{40}$ & 0.004 & 1.00 \\
\hline PTF 10tel & IIn & 260.37782 & +48.12983 & 17 & -18.5 & 0.035 & 55450 & 2.34 & $<7.2 \times 10^{41}$ & 0.1 & 1.00 \\
\hline SN 2011iw & IIn & 353.70083 & -24.75044 & $<40$ & $-18.1:$ & 0.023 & 55895: & 1.61 & $<1.0 \times 10^{41}$ & 0.02 & 1.00 \\
\hline SN $2005 \mathrm{db}$ & IIn & 10.36163 & +25.49767 & $<18$ & $-16.8:$ & 0.0153 & 53570: & 4.17 & $\sim 5.3 \times 10^{40}$ & 0.04 & 0.00 \\
\hline SN 2005av & IIn & 311.15658 & -68.75294 & $<19$ & $-17.8:$ & 0.0104 & 53453: & 4.85 & $<8.1 \times 10^{39}$ & 0.02 & 1.00 \\
\hline SN 20031o & IIn & 54.27133 & -5.03814 & $\ldots$ & $-15.8:$ & 0.0079 & 53005: & 4.87 & $<6.3 \times 10^{39}$ & 0.01 & 1.00 \\
\hline $\mathrm{SN} 2002 \mathrm{fj}$ & IIn & 130.18792 & -4.12736 & $<90$ & -18.5 & 0.0145 & 52532 & 3.12 & $<4.9 \times 10^{40}$ & 0.007 & 1.00 \\
\hline
\end{tabular}

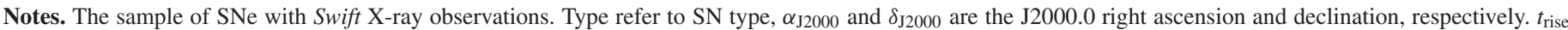

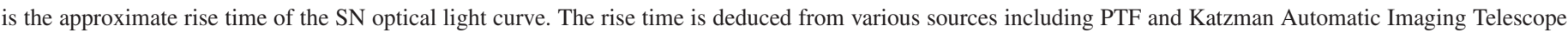

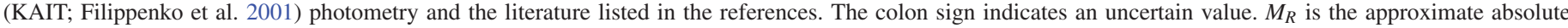

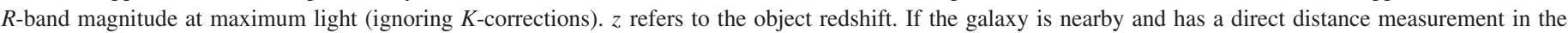

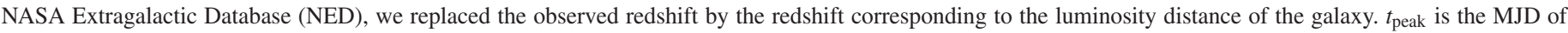

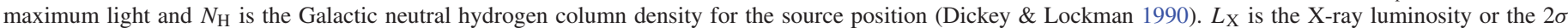

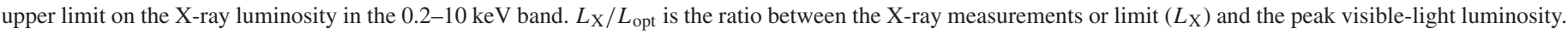

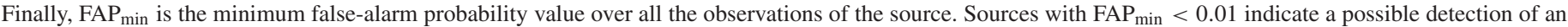

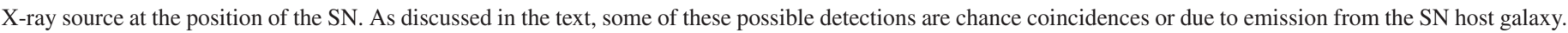

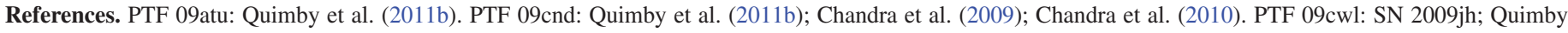

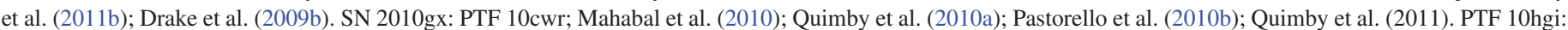

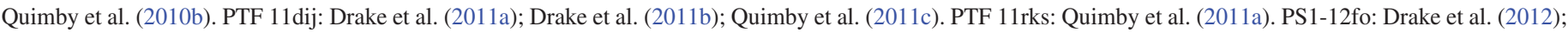

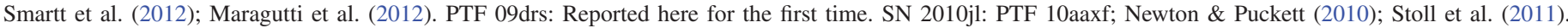

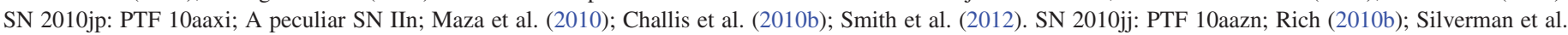

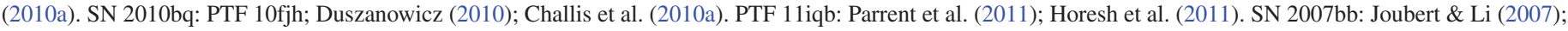

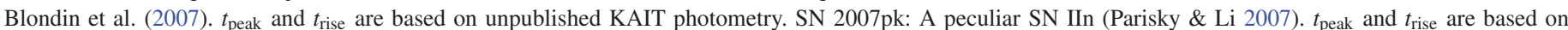

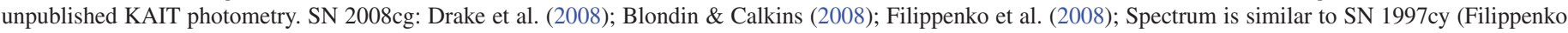

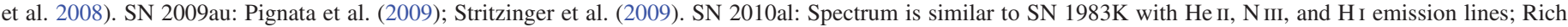

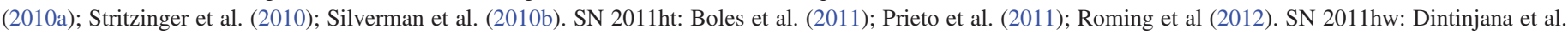

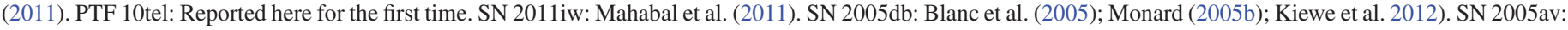
Monard (2005a); Salvo et al. (2005). SN 2003lo: Puckett et al. (2004); Matheson et al. (2004). SN 2002fj: Monard \& Africa (2002); Hamuy (2002).

et al. 2012) that may show a dip in the light curve followed by rebrightening. Moriya \& Maeda (2012) interpret the dip as an increase in the opacity due to ionization of the massive shell/CSM as it interacts with the ejecta.

Our sample, presented in Table 1, consists of eight SLSN-I objects, 19 SNe IIn, and a single SN Ibn. We note that the spectra of SNe having PTF names, as well as some other SNe, are available online from the WISeREP ${ }^{18}$ Web site (Yaron \& Gal-Yam 2012).

\footnotetext{
18 Weizmann Interactive Supernova (data) REPository; http://www.weizmann.ac.il/astrophysics/wiserep/.
}

\section{OBSERVATIONS}

For each Swift/XRT image of an SN, we extracted the number of X-ray counts in the $0.2-10 \mathrm{keV}$ band within an aperture of 7".2 (3 pixels) radius centered on the $\mathrm{SN}$ position. We chose a small aperture in order to minimize any host-galaxy contamination. We note that this aperture contains $\sim 37 \%$ of the source flux (Moretti et al. 2004). The background count rates were estimated in annuli around each $\mathrm{SN}$, with an inner (outer) radius of $50^{\prime \prime}\left(100^{\prime \prime}\right)$. For each SN that has Swift/XRT observations, we searched for Chandra observations. The Chandra observations were analyzed in a similar manner with an extraction aperture 
Table 2

Swift/XRT X-Ray Measurements

\begin{tabular}{|c|c|c|c|c|c|c|c|c|c|}
\hline \multirow[t]{2}{*}{ Name } & \multicolumn{2}{|c|}{$t-t_{\text {peak }}$} & \multirow{2}{*}{$\begin{array}{l}\text { Exp. } \\
\text { (s) }\end{array}$} & \multirow[b]{2}{*}{$\left(\right.$ counts ks ${ }^{-1}$ ) } & \multirow[b]{2}{*}{$\left(\right.$ counts ks ${ }^{-1}$ ) } & \multirow[b]{2}{*}{$\left(\right.$ counts ks $\left.{ }^{-1}\right)$} & \multirow[b]{2}{*}{ (counts ks ${ }^{-1}$ ) } & \multirow[t]{2}{*}{ FAP } & \multirow{2}{*}{$\begin{array}{c}L_{\mathrm{X}} \\
\left(\mathrm{erg} \mathrm{s}^{-1}\right)\end{array}$} \\
\hline & $\begin{array}{l}\text { (Start } \\
\text { Day) }\end{array}$ & $\begin{array}{l}\text { (End } \\
\text { Day) }\end{array}$ & & & & & & & \\
\hline PTF 09atu & 2.7 & $\ldots$ & 4858.0 & -0.02 & $\ldots$ & $\ldots$ & 0.62 & 1.00 & $<1.9 \times 10^{44}$ \\
\hline \multirow{11}{*}{ PTF 09cnd } & -17.6 & $\ldots$ & 3441.8 & -0.02 & $\ldots$ & $\ldots$ & 0.87 & 1.00 & $<5.4 \times 10^{43}$ \\
\hline & -13.7 & $\cdots$ & 3557.2 & -0.01 & $\cdots$ & $\cdots$ & 0.84 & 1.00 & $<5.3 \times 10^{43}$ \\
\hline & -10.5 & $\ldots$ & 3273.1 & -0.02 & $\ldots$ & $\ldots$ & 0.92 & 1.00 & $<5.7 \times 10^{43}$ \\
\hline & -5.9 & $\cdots$ & 3997.8 & -0.02 & $\cdots$ & $\cdots$ & 0.75 & 1.00 & $<4.7 \times 10^{43}$ \\
\hline & -2.2 & $\ldots$ & 2232.1 & -0.05 & $\ldots$ & $\ldots$ & 1.34 & 1.00 & $<8.4 \times 10^{43}$ \\
\hline & 4.5 & $\ldots$ & 2980.6 & -0.03 & $\ldots$ & $\ldots$ & 1.01 & 1.00 & $<6.3 \times 10^{43}$ \\
\hline & 18.3 & $\ldots$ & 2026.8 & -0.01 & $\ldots$ & $\ldots$ & 1.48 & 1.00 & $<9.2 \times 10^{43}$ \\
\hline & 27.9 & $\ldots$ & 1910.6 & -0.02 & $\ldots$ & $\cdots$ & 1.57 & 1.00 & $<9.8 \times 10^{43}$ \\
\hline & -17.6 & -2.2 & 16501.9 & -0.02 & $\ldots$ & $\ldots$ & 0.18 & 1.00 & $<1.1 \times 10^{43}$ \\
\hline & 4.5 & 27.9 & 6917.9 & -0.02 & $\cdots$ & $\cdots$ & 0.43 & 1.00 & $<2.7 \times 10^{43}$ \\
\hline & -17.6 & 27.9 & 23419.8 & -0.02 & $\cdots$ & $\cdots$ & 0.13 & 1.00 & $<8.0 \times 10^{42}$ \\
\hline
\end{tabular}

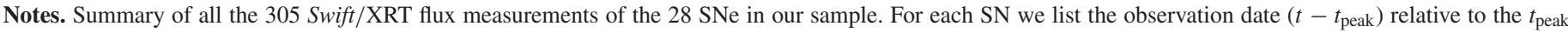

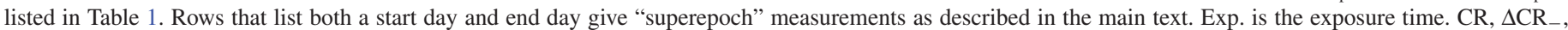

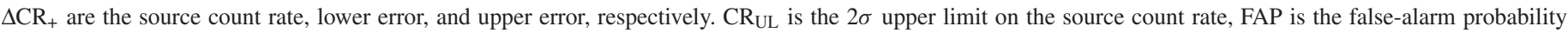

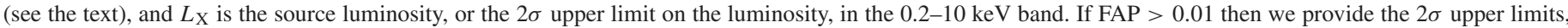
otherwise the measurements are given along with the uncertainties.

(This table is available in its entirety in a machine-readable form in the online journal. A portion is shown here for guidance regarding its form and content.)

of $2^{\prime \prime}$ and background annuli with an inner (outer) radius of $15^{\prime \prime}\left(40^{\prime \prime}\right)$. All of the Swift/XRT X-ray measurements are listed in Table 2 (the full table is available in the online version). In addition, in Table 2, for each object we give the count rate in up to four superepochs: (1) all of the observations taken prior to maximum light, or discovery date if time of maximum light is not known; (2) all of the observations taken between maximum light and 300 days after maximum light; (3) all of the observations taken more than 300 days after maximum light; and (4) all of the observations at the position of the SN.

In each epoch, and superepoch, we also provide an estimator for the false-alarm probability (FAP), which is the probability that the $\mathrm{X}$-ray counts are due to the X-ray background rather than a source. This probability is estimated as 1 minus the Poisson cumulative distribution to get a source count rate smaller than the observed count rate, assuming the expectancy value of the Poisson distribution equal to the background counts. We note that the median background counts in our background annuli is six counts (within an exposure). However, the background annuli area is about 340 times larger than the photometric aperture area. Therefore, the contribution of the errors in the background to the uncertainty in the source counts is negligible. We note that in some cases X-ray emission from the host galaxy will tend to produce some seemingly significant, but actually "false alarm" detections under these assumptions. Furthermore, many nuclear X-ray sources like active galactic nuclei (AGNs), as well as X-ray sources in general, tend to significantly vary with time (e.g., González-Martín \& Vaughan 2012). This fact makes it very hard to distinguish between X-ray emission from an SN and its host galaxy. An example for such confusion is demonstrated in the case of SN 2007pk discussed in this paper.

In cases in which FAP $\leqslant 0.01$, we also estimated the $2 \sigma$ upper limit on the count rate (Gehrels 1986). The count-rate measurements or upper limits are converted to luminosity in the $0.2-10 \mathrm{keV}$ band using the PIMMS ${ }^{19}$ web tool and assuming that (1) the aperture in which we extracted the source photometry

\footnotetext{
19 http://cxc.harvard.edu/toolkit/pimms.jsp
}

contains $37 \%$ of the source photons (Moretti et al. 2004); (2) Galactic neutral hydrogen column density at the position of the sources as listed in Table 1 (Dickey \& Lockman 1990); (3) a spectrum of $N_{\mathrm{ph}}(E) \propto E^{-0.2}$, where $N(E)$ has units of photons $\mathrm{cm}^{-2} \mathrm{~s}^{-1}$ (motivated in Section 4); and (4) the luminosity distance to each $\mathrm{SN}$ calculated using the redshift listed in Table 1 together with $H_{0}=70.4 \mathrm{~km} \mathrm{~s}^{-1} \mathrm{Mpc}^{-1}$, $\Omega_{m}=0.268, \Omega_{\Lambda}=0.716$, and $\Omega_{K}=0.986$ (the third-year WMAP+SNLS cosmology; Spergel et al. 2007).

Several objects listed in Table 2 show count rates which may deviate from zero. Here we discuss the observations of all seven sources that have FAP $\leqslant 0.01$ in at least one of the epochs or superepochs. We note that Table 2 contains 305 epochs and superepochs; therefore, we expect about three random false detections. Interpretation of these observations is discussed in Section 5.

SN 2010jl/PTF 10aaxf (Figure 1). This SN has a large number of Swift/XRT observations, as well as Chandra/ACIS-S observations in five epochs (Chandra et al. 2012b), of which three are public (PIs: D. Pooley; Tremonti). The host, SDSSJ094253.43+092941.9, is an irregular star-forming galaxy. The binned Swift/XRT and Chandra light curves, as well as the PTF $R$-band light curve, are presented in Figure 1.

$S N$ 2006jc (Figure 2). This is the only SN in our sample that belongs to the rare class of Type Ibn SNe. SN 2006jc has a large number of Swift/XRT and Chandra observations. The SN is detected on multiple epochs and its X-ray light curve is presented in Figure 2 (see also Immler et al. 2008). It was detected in X-rays soon after maximum optical light and reached a maximum $X$-ray luminosity of about $1.5 \times 10^{40} \mathrm{erg} \mathrm{s}^{-1}$ at $\sim 100$ days after maximum optical light, $\gtrsim 6$ times the SN rise time. SN 2006jc was observed by Chandra on several occasions. We reduced a $55 \mathrm{ks}$ Chandra observation with 306 photons at the SN location taken 87 days after maximum light. Given the limited number of photons we did not attempt to fit complex models. We found that the spectrum is well fitted by an $N_{\mathrm{ph}}(E) \propto E^{-0.2}$ power law and with negligible absorbing column density. The spectra and the best-fit model are presented 


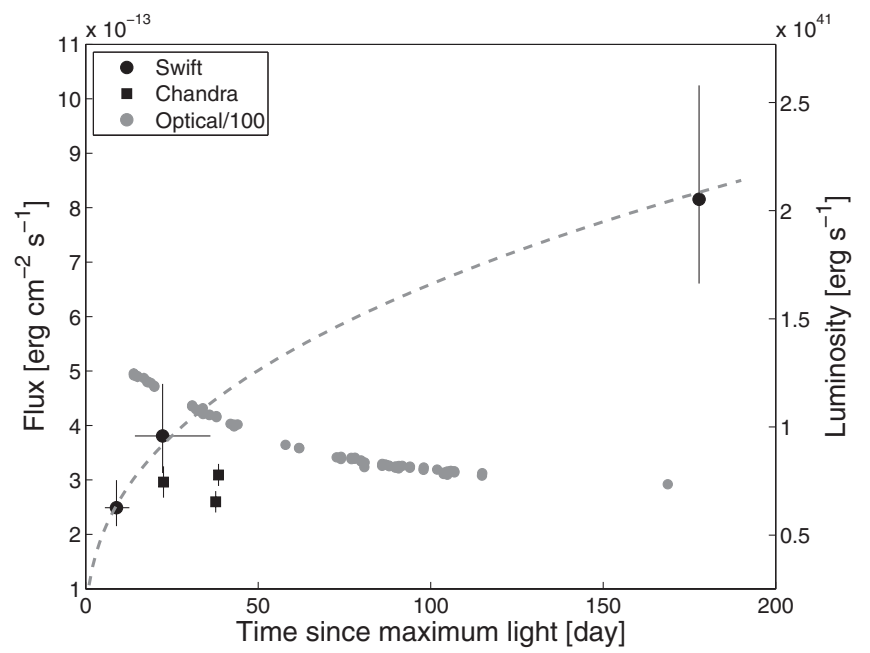

Figure 1. Swift (circles) and Chandra (squares) X-ray light curves extracted at the position of SN 2010jl. The unabsorbed flux was calculated using PIMMS assuming a Galactic column density of $N_{\mathrm{H}}=3.05 \times 10^{20} \mathrm{~cm}^{-2}$, and a $N_{\text {ph }}(E) \propto E^{-0.2}$ power-law spectrum. We note that the Chandra observations show a possible extended source near the SN location. This additional source may contaminate the Swift/XRT measurements and can explain the small discrepancy between Chandra and Swift/XRT. Alternatively, the discrepancy between the Chandra and Swift light curves can be explained if the X-ray spectrum is harder or $N_{\mathrm{H}}$ is larger than we assumed. We note that for $N_{\mathrm{H}}$ which is a factor of 1000 larger than the Galactic value, the unabsorbed Swift (Chandra) flux will be about 5.2 (7.2) times larger. For reference, the gray circles show the PTF $R$-band luminosity of this SN scaled by 0.01 . The PTF $R$-band luminosity was calibrated using the method described by Ofek et al. (2012a) and calibration stars listed by Ofek et al. (2012b).

in Figure 3. Regardless of the exact spectral shape, the spectrum is hard. Marginalizing over all the free parameters, we find a $2 \sigma$ upper limit of $N_{\mathrm{H}}<1.26 \times 10^{21} \mathrm{~cm}^{-2}$, in excess of the Galactic column density.

SN 2011ht (Figure 4). This SN took place about $21^{\prime \prime}$ from the center of UGC 5460. It was observed on multiple epochs using Swift/XRT, and Roming et al. (2012) reported a detection of an X-ray source at the SN position. The binned Swift/XRT $\mathrm{X}$-ray light curve of this $\mathrm{SN}$ is shown in Figure 4. Apparently the light curve rises, peaks $\sim 40$ day after maximum optical light, and then declines. However, the uncertainties in the flux measurements are large and the light curve is consistent with being flat (i.e., a best-fit flat model gives $\chi^{2} /$ dof $=1.23 / 4$ ). Moreover, recently Pooley (2012) reported on a $9.8 \mathrm{ks}$ Chandra observation $^{20}$ of this SN. He argued that the emission detected by Roming et al. (2012), which is shown in Figure 4, is from a nearby source found 4".7 from the SN location.

SN 2010al (Figure 5). This SN was found 12" from the center of the spiral galaxy UGC 4286. It was observed on multiple epochs using Swift/XRT with a total integration time of $35 \mathrm{ks}$, and is detected in the combined image with a mean luminosity of $\sim 7 \times 10^{39} \mathrm{erg} \mathrm{s}^{-1}$. Figure 5 presents the binned Swift/XRT light curve. Although the light curve peaks around 30 days after maximum light, it is consistent with being flat (i.e., a best-fit flat model gives $\chi^{2} /$ dof $=0.15 / 2$ ).

$S N 2005 d b$. This SN was observed on three epochs, about two years after maximum light (676-695 days), using Swift/ XRT. The combined image, with an exposure time of $13.6 \mathrm{ks}$, shows a faint source (five photons) with an FAP of $2 \times 10^{-4}$ per trial. However, given the fact that we have 305 observations, the FAP over all the trials is only about 0.06. Using the

20 At the time of the writing this paper, this observation was proprietary.

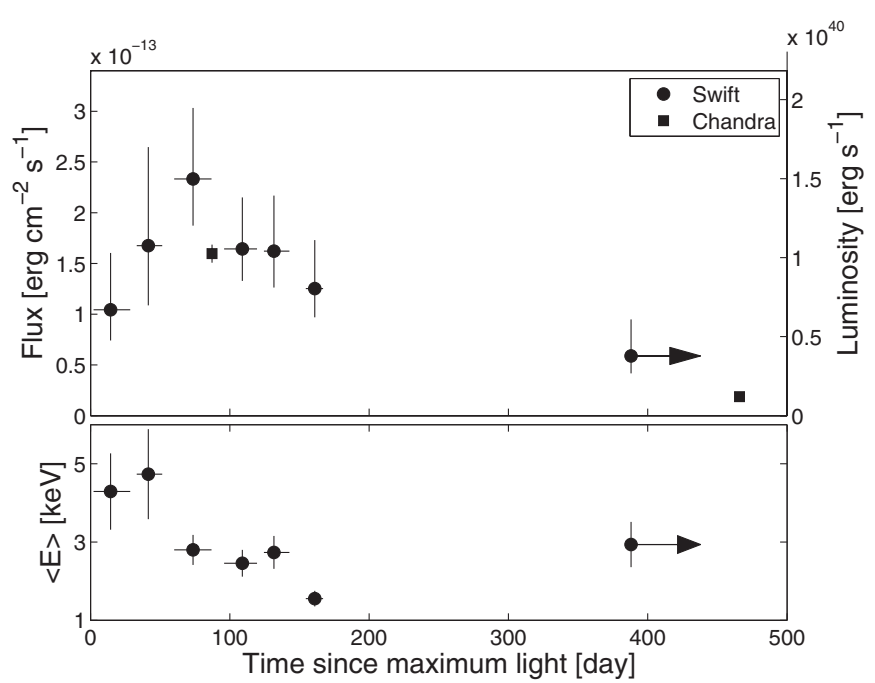

Figure 2. Upper panel: the Swift (circles) and Chandra (squares) X-ray light curve extracted at the position of SN 2006jc. The unabsorbed flux was calculated using PIMMS assuming a Galactic column density of $N_{\mathrm{H}}=1.0 \times 10^{20} \mathrm{~cm}^{-2}$, and a $N_{\mathrm{ph}}(E) \propto E^{-0.2}$ power-law spectrum. Lower panel: the mean photon energy of the Swift/XRT X-ray observations in the $0.2-10 \mathrm{keV}$ band as a function of time. A version of this plot is shown by Immler et al. (2008).

Galactic column density (Table 1) and assuming a power-law spectrum $N_{\mathrm{ph}}(E) \propto E^{-0.2}$, the unabsorbed flux is $\left(1.97_{-0.72}^{+1.7}\right) \times$ $10^{40} \mathrm{erg} \mathrm{s}^{-1}$. Chandra observed this target twice at 722.7 and 1051.4 days after maximum light (PI: D. Pooley), with integrations of 3.0 and $5.0 \mathrm{ks}$, respectively. Using the same assumptions as above, we put a $2 \sigma$ upper limit on the unabsorbed flux of $1.4 \times 10^{40} \mathrm{erg} \mathrm{s}^{-1}$ for both epochs. To conclude, given the uncertainties, the Chandra upper limits are consistent with the possible Swift detection. However, given that this source has a single detection, we cannot firmly conclude that the detection is real.

PTF 1 liqb. This SN has multi-epoch Swift/XRT observations taken between about -13 and 28 days relative to maximum light. The $\mathrm{SN}$ is detected in a single epoch $\sim 24$ days after maximum light, with an FAP of $1.4 \times 10^{-3}$ per trial. However, given that we are reporting 305 individual X-ray observations, the FAP over all the trials is 0.45 . The $\mathrm{SN}$ was not detected at the last epoch, 28 days after maximum light.

SN 2007pk (Figure 6). This SN has a large number of Swift/XRT observations, as well as a Chandra observation (PI: D. Pooley). Immler et al. (2007) reported a tentative detection in the images taken between MJD 54417.09 and 54420.04. The light curve of the source extracted at the SN position is shown in Figure 6. The light curve shows a brightening, with a peak around MJD 54461, and a full width at halfmaximum intensity of about 40 days. However, the $\mathrm{SN}$ is about $7^{\prime \prime}$ from the center of the spiral host galaxy, NGC 579, and the centroids of the Swift/XRT positions in individual exposures are clustered around the galaxy nucleus, rather than at the SN position. We note that emission from the center of NGC 579 is clearly detected in the Chandra observation and that there is some emission at the source position. However, the latter may be due to diffuse emission from NGC 579. Therefore, without conclusive evidence that the emission is from the $\mathrm{SN}$, here we assume that the observed flare as well as the quiescent $\mathrm{X}$-ray emission from the position of the source is due to AGN activity in NGC 579. In Table 1, we adopted an upper limit on the X-ray luminosity of SN 2007pk, which is based on the 


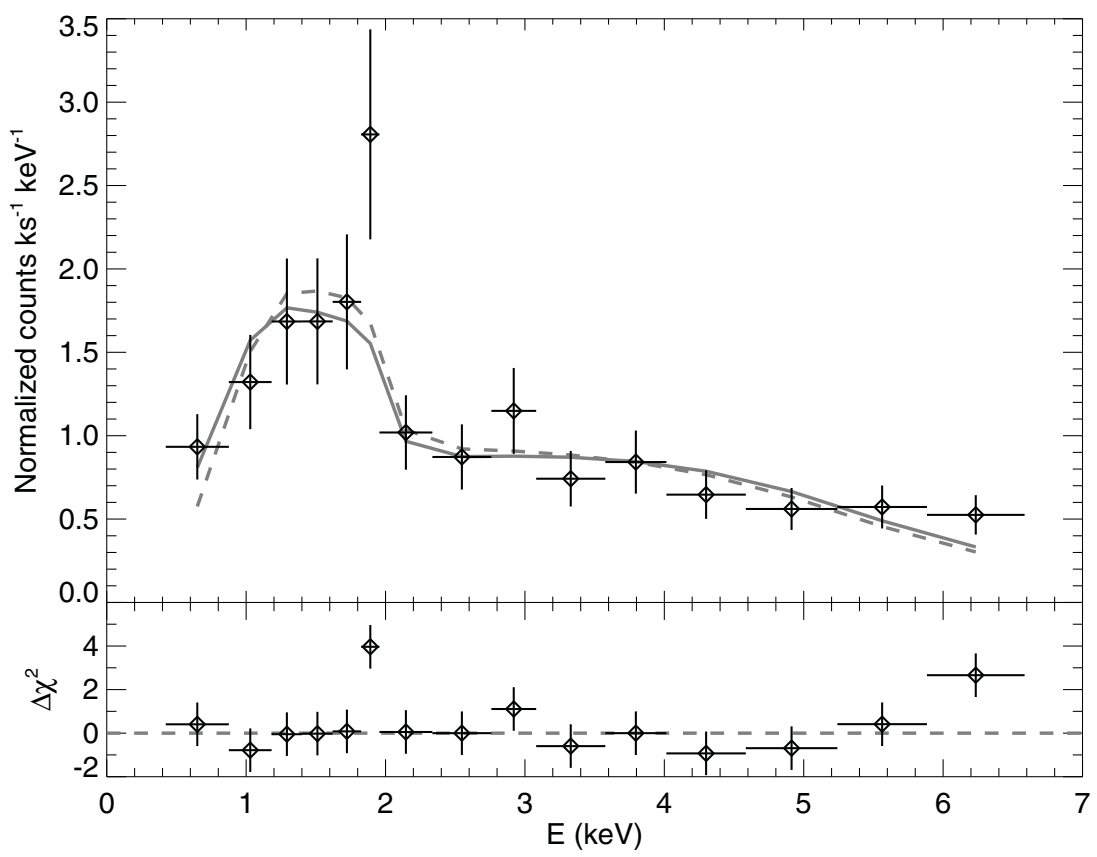

Figure 3. Spectrum of SN 2006jc, as observed 87 days after maximum optical light, near or at peak X-ray luminosity. The top panel shows the binned X-ray data (points) with errors $(1 \sigma)$ along with the best-fit model, a photon index $\Gamma=0.24_{-0.16}^{+0.22}$ (90\% confidence) power law, where $N_{\text {ph }} \propto E^{-\Gamma}$, and with negligible line-of-sight absorption. The dashed line shows the constrained best fit that has maximal $N_{\mathrm{H}}\left(90 \%\right.$ confidence, $1.37 \times 10^{21} \mathrm{~cm}^{-2}$ ). The bottom panel shows the $\Delta \chi^{2}$ residuals to the best fit. The fit is acceptable, with $\chi^{2}=11.75$ for 12 degrees of freedom $\left(p_{\text {null }}=0.47\right)$.

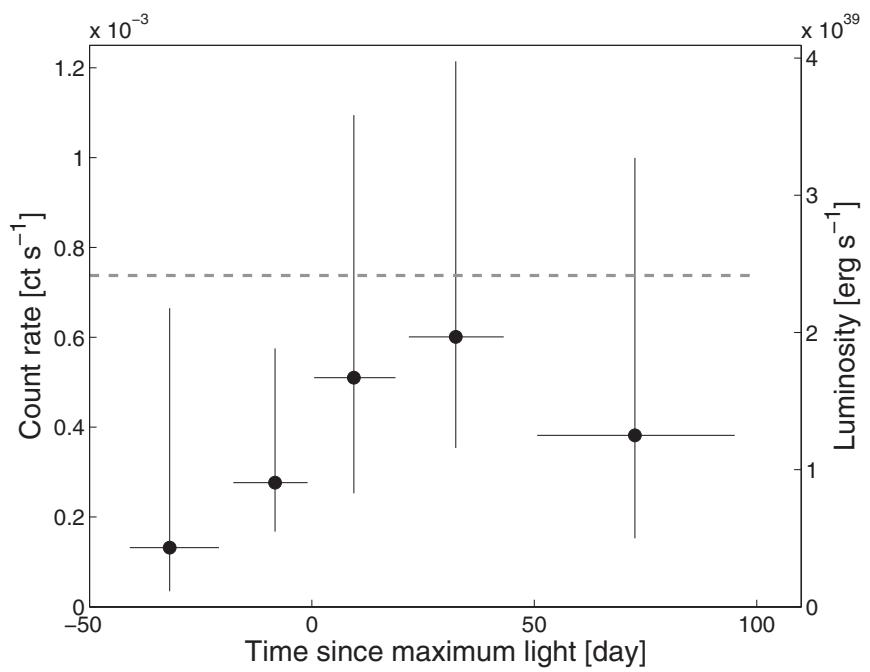

Figure 4. Unabsorbed Swift/XRT light curve extracted at the position of SN $2011 \mathrm{ht}$ in the $0.2-10 \mathrm{keV}$ band, corrected for the aperture size and assuming $N_{\mathrm{H}}=7.8 \times 10^{19} \mathrm{~cm}^{-2}$. The dashed gray line represents the $2 \sigma$ upper limit on the flux from two combined Swift/XRT observations obtained 1649 and 1405 days prior to the SN maximum light.

average luminosity observed from the direction of the source, presumably due to AGN activity.

\section{THE MODEL}

Several recent works discuss the possibility of detecting $\mathrm{X}$-ray emission from SN collisionless shocks in optically thick wind environments (Katz et al. 2011; Murase et al. 2011; Chevalier \& Irwin 2012; Svirski et al. 2012). Here, we review the main processes relevant to shock breakout in wind-profile CSM (Section 4.1), emission from collisionless shocks including the importance of bound-free absorption (Section 4.2), and the

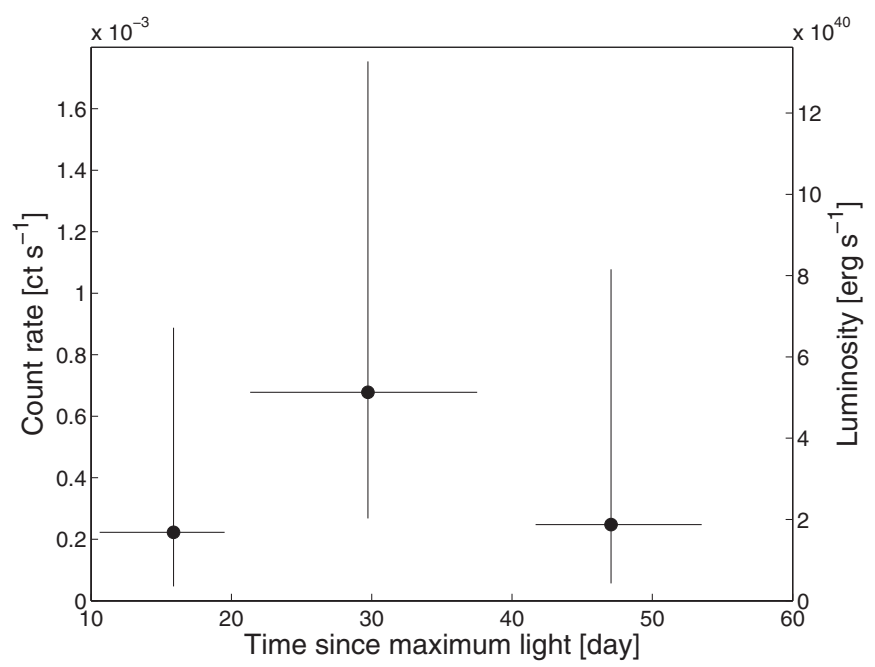

Figure 5. Unabsorbed Swift/XRT light curve extracted at the position of SN 2010al in the $0.2-10 \mathrm{keV}$ band, corrected for the aperture size and assuming $N_{\mathrm{H}}=3.92 \times 10^{20} \mathrm{~cm}^{-2}$.

possibility of detecting radio emission (Section 4.3). In Section 5 we discuss our observations in the context of this model.

\subsection{Shock-breakout Conditions in Wind-profile CSM}

Here, we assume that the CSM around the progenitor has a wind-density profile $\rho=K r^{-2}$, where $K \equiv M /\left(4 \pi v_{w}\right)$ is the mass-loading parameter, $\dot{M}$ is the progenitor mass-loss rate, and $v_{w}$ is the progenitor wind speed. The Thomson optical depth, $\tau$, due to an ionized progenitor wind between the observer and a spherical surface at radius $r$ from the star center is

$$
\begin{aligned}
\tau & \approx \frac{\kappa \dot{M}}{4 \pi v_{\mathrm{w}} r} \\
& \approx 170 \kappa_{0.34} \dot{M}_{0.1} v_{\mathrm{w}, 10}^{-1} r_{15}^{-1} .
\end{aligned}
$$




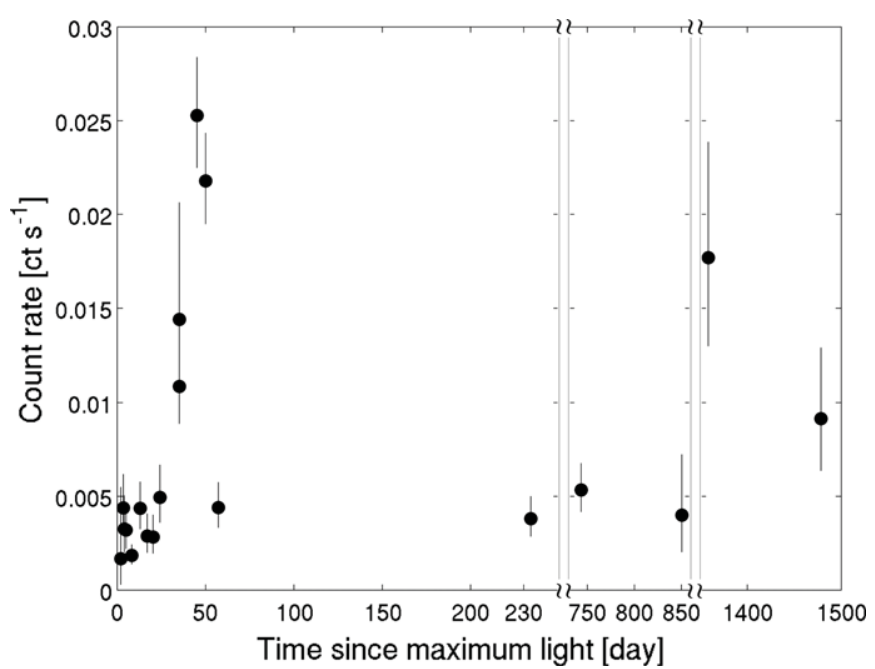

Figure 6. X-ray light curve, corrected for aperture size, extracted at the position SN 2007pk. The emission, and flare, are likely due to AGN activity in the host galaxy NGC 579 .

Here $\kappa_{0.34}$ is the opacity in units of $0.34 \mathrm{~cm}^{2} \mathrm{~g}^{-1}, \dot{M}_{0.1}$ is the mass-loss rate in units of $0.1 M_{\odot} \mathrm{yr}^{-1}, v_{\mathrm{w}, 10}$ is the wind speed in units of $10 \mathrm{~km} \mathrm{~s}^{-1}$, and $r_{15}$ is the radius in units of $10^{15} \mathrm{~cm}$. We note that this relation is correct up to a factor of order unity (see Balberg \& Loeb 2011). The photons in the radiation-dominated and radiation-mediated shock from the SN explosion break out when $\tau \approx c / v_{\mathrm{s}}$ (e.g., Weaver 1976; Ofek et al. 2010), where $c$ is the speed of light and $v_{\mathrm{s}}$ is the shock velocity. At this optical depth, the photon diffusion time becomes shorter than the hydrodynamical timescale (i.e., $r / v_{\mathrm{s}}$ ) and the photons can diffuse outward faster than the ejecta. Therefore, the condition for the shock breakout to take place in a steady-wind environment $(w=2)$ is

$$
\frac{\dot{M}_{0.1}}{v_{\mathrm{w}, 10}} \gtrsim 1.2 \times 10^{-6} \tau_{30} r_{\odot} \kappa_{0.34}^{-1}\left(0.01 M_{\odot} \mathrm{yr}^{-1} \mathrm{~km}^{-1} \mathrm{~s}\right)
$$

where $\tau_{30}$ is the Thomson optical depth in units of 30 and $r_{\odot}$ is the radius in units of the solar radius.

Figure 7 shows the radius versus the mass-loading parameter at which $\tau \approx 30$ (Equation (2); i.e., $v_{\mathrm{s}}=10^{4} \mathrm{~km} \mathrm{~s}^{-1}$; solid black line). For example, this plot suggests that the critical mass-loading, above which the shock will break out in the wind environment, is $\sim 6 \times 10^{-4} M_{\odot} \mathrm{yr}^{-1} v_{\mathrm{w}, 10}^{-1}$ for a $500 R_{\odot}$ red supergiant. Assuming $v_{\mathrm{s}}=10^{4} \mathrm{~km} \mathrm{~s}^{-1}$, systems found above the solid line will have a shock breakout in a wind profile (the two cases discussed by Chevalier \& Irwin 2011). Objects found in regions below the $\tau \approx 2 / 3$, black dashed-dotted line, will have a shock breakout within the stellar surface and the wind will not influence the diffusion of energy from the shock breakout. Finally, systems below the solid line $(\tau \approx 30)$ and above the dashed-dotted line $(\tau \approx 2 / 3)$ will have a shock breakout below the stellar surface, but the wind can play a role in the diffusion of the shock energy (e.g., Nakar \& Sari 2010).

We note that, in order to form an SN IIn, we require optically thin material, which is ionized by the SN radiation field. Therefore, we speculate that SNe IIn can be found below and above the $\tau \approx 2 / 3$ line, and that not all SNe IIn are powered by shock breakout in dense CSM environments.

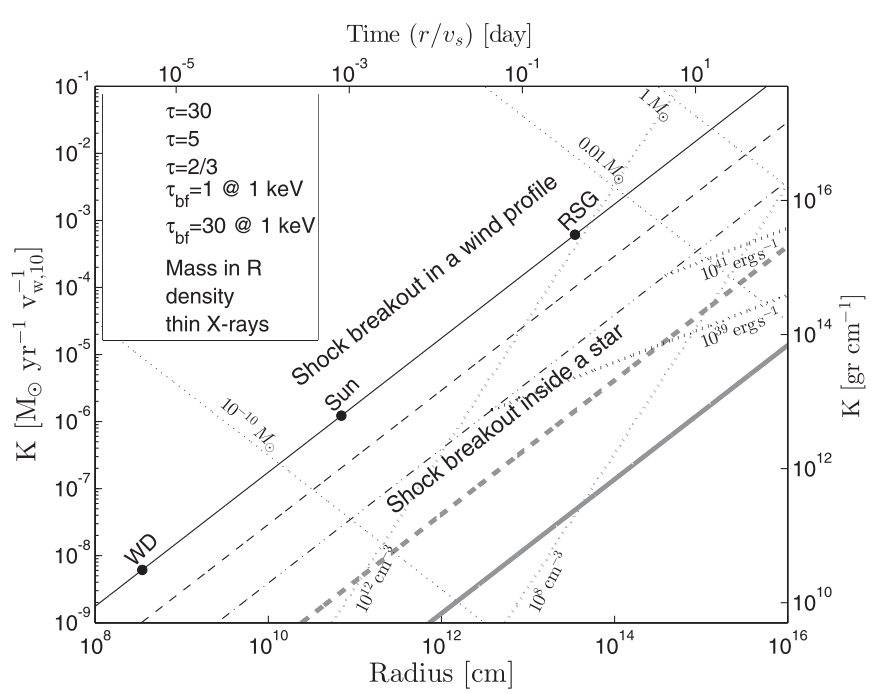

Figure 7. Various regions in the radius vs. wind mass-loading parameter phase space, assuming $w=2$. As indicated in the legend the solid black line represents Thomson optical depth, $\tau=30$ (i.e., shock breakout with $v_{\mathrm{s}}=10^{4} \mathrm{~km} \mathrm{~s}^{-1}$ ). $\tau=5$ and $\tau=2 / 3$ are marked with the dashed black, and dashed-dotted black lines, respectively. The dotted black lines represent the integral of mass inside a given radius (i.e., $\int_{0}^{r} 4 \pi r^{2} K r^{-2} d r=4 \pi K r$ ). The gray lines show constant bound-free absorption at a given energy (see legend). Note that $\tau_{\mathrm{bf}}$ is calculated using the relation given in Morrison \& McCammon (1983), rather than the approximate relation provided in Equation (5). The dotted gray lines represent lines of constant density, while the heavy dotted black lines shows constant optically thin X-ray emission (see the text). For reference, the radii of red supergiant (RSG, $500 R_{\odot}$ ), the Sun and a massive white dwarf (WD, $0.005 R_{\odot}$ ) are marked in circles on the $\tau=30$ line. See the text for discussion.

\subsection{X-Ray Emission from Collisionless Shocks}

Katz et al. (2011) showed that if the shock width $(\Delta r)$ at radius $r$ is on the order of $r$ (i.e., the Thomson optical depth varies on scales on the order of the radius $r$ ), the radiationmediated and radiation-dominated shock will transform into a collisionless shock, and hard X-ray photons will be generated. It is reasonable to assume that some of this energy will be produced in the $1-10 \mathrm{keV} \mathrm{X}$-ray regime. We note that the exact spectrum was not calculated self-consistently and, therefore, is not known.

Chevalier \& Irwin (2012) and Svirski et al. (2012) showed that, during the first several shock-breakout timescales after the shock breakout, the optical depth is too large for the hard $\mathrm{X}$-rays to escape. They found that the most efficient processes in blocking the hard X-rays $(\sim 100 \mathrm{keV})$ are likely (1) cooling of the electrons by inverse-Compton scattering on soft photons generated by the pre-shocked material and (2) Compton scattering. On average, for each Compton scattering, the photon loses a fraction of its energy which is comparable to $4 k_{\mathrm{B}} T_{\mathrm{e}} /\left(m_{\mathrm{e}} c^{2}\right)$ (e.g., Lang 1999). Here $k_{\mathrm{B}}$ is the Boltzmann constant, $T_{\mathrm{e}}$ is the electron temperature, and $m_{\mathrm{e}}$ is the electron mass. Svirski et al. (2012) argued that when the Thomson optical depth declines to $\tau \approx 5$, the hard $\mathrm{X}$-ray emission $(\sim 100 \mathrm{keV})$ will be Comptonized to the 1-10 keV X-ray band and may diffuse out of the CSM. Since the optical depth in a wind profile $(w=2)$ decreases as $r^{-1}$ (Equation (1)), the collisionless-shock signature will be observable in the X-ray regime only when the optical depth decreases by an order of magnitude (i.e., the shock radius increases by an order of magnitude). Given that the shock velocity falls as $t^{-1 / 5}$, they argued that this should happen roughly between 10 and 50 times the shock-breakout timescale. For reference, we show the $\tau=5$ line in Figure 7 (dashed line, 
assuming $v_{\mathrm{s}}=10^{4} \mathrm{~km} \mathrm{~s}^{-1}$ ). Such late-time emission may be relevant only if the collisionless shock is still important at late times and if the Comptonization remains the dominant process.

Chevalier \& Irwin (2012) consider the effect of boundfree absorption. Since above $0.5 \mathrm{keV}$, metals with a high ionization potential dominate the bound-free absorption, full ionization is required in order to avoid absorption by this process. They argued that an ionization parameter of $\sim 1000$ is needed to achieve full ionization (including the metal atoms' inner electrons). They estimate that full ionization is achieved for shocks with velocity $v_{\mathrm{s}} \gtrsim 10^{4} \mathrm{~km} \mathrm{~s}^{-1}$. In order to estimate the effect of bound-free absorption, we need to evaluate the density of the CSM.

Assuming a hydrogen-rich material, the particle density in a wind profile is given by

$$
\begin{aligned}
n & =\frac{1}{\left\langle\mu_{\mathrm{p}}\right\rangle} \frac{\dot{M}}{4 \pi m_{\mathrm{p}} v_{\mathrm{w}} r^{2}} \\
& \approx 3.02 \times 10^{11} \frac{1}{\left\langle\mu_{\mathrm{p}}\right\rangle} \dot{M}_{0.1} v_{\mathrm{w}, 10}^{-1} r_{15}^{-2} \mathrm{~cm}^{-3}
\end{aligned}
$$

where $\left\langle\mu_{\mathrm{p}}\right\rangle$ is the mean number of protons per particle (mean molecular weight). For our order-of-magnitude calculation, we assume $\left\langle\mu_{\mathrm{p}}\right\rangle=1$. In a wind profile, the column density between the radius $r$ and the observer is

$$
N \approx 3.02 \times 10^{26} \dot{M}_{0.1} v_{\mathrm{w}, 10}^{-1} r_{15}^{-1} \mathrm{~cm}^{-2} .
$$

Assuming the gas in the pre-shocked wind is neutral, the boundfree optical depth in the $0.03-10 \mathrm{keV}$ region is roughly given by (e.g., Behar et al. 2011) ${ }^{21}$

$$
\begin{aligned}
\tau_{\mathrm{bf}} & =N \sigma(E) \\
& \approx 3 \times 10^{4} \dot{M}_{0.1} v_{\mathrm{w}, 10}^{-1} r_{15}^{-1} E_{1}^{-2.5},
\end{aligned}
$$

where $\sigma(E)$ is the bound-free cross section as a function of energy $E$ and $E_{1}$ is the energy in $\mathrm{keV}$. This approximation is valid when the material is neutral. However, since above $\sim 0.5 \mathrm{keV}$ metals with a high ionization potential dominate the absorption, this formula is still valid, to an order of a magnitude, above $0.5 \mathrm{keV}$ when some (or even one) of the inner electrons of the metals are bound. In Figure 7 we show the lines at which $\tau_{\text {bf }} \approx 1$ at $1 \mathrm{keV}$ and $10 \mathrm{keV}$, and at which $\tau_{\text {bf }} \approx 30$ at $1 \mathrm{keV}$. Comparison of Equations (1) and (5) suggests that at the time of shock breakout the bound-free cross section in the $\sim 0.5-8 \mathrm{keV}$ range is larger than the Thomson cross section. We note that this may modify the properties of the shock breakout and its spectrum. Moreover, the $\tau_{\mathrm{bf}}=1$ line at $1 \mathrm{keV}$ is located far below the $\tau=5$ line. This suggests that if the pre-shocked wind is not completely ionized (e.g., $v_{\mathrm{s}} \lesssim 10^{4} \mathrm{~km} \mathrm{~s}^{-1}$ ), then soft $(\lesssim 1 \mathrm{keV}) \mathrm{X}$-ray emission is not expected in the simple case of a spherically symmetric wind $(w=2)$ profile, even at late times. Moreover, the $\tau_{\mathrm{bf}}=1$ at $10 \mathrm{keV}$ line is located slightly below the $\tau=5$ line. Therefore, bound-free absorption is likely important even at late stages. This may indicate that $\sim 10 \mathrm{keV}$ $\mathrm{X}$-rays may escape the wind on a timescale somewhat longer than suggested by Svirski et al. (2012), and that observations at energies above $10 \mathrm{keV}$ may be more effective (e.g., by the NUSTAR mission; Harrison et al. 2010).

All of the order-of-magnitude calculations presented so far assume a wind-density profile with $w=2$. However, we note

\footnotetext{
21 This approximation deviates by a factor of two from a more accurate
} calculation (e.g., Morrison \& McCammon 1983). that if the CSM profile falls faster than $r^{-2}$, or alternatively if the wind is not spherically symmetric or is clumpy, then the column density may fall faster than $r^{-1}$ in some directions (e.g., Equation (4)), and it will enable the X-rays to escape earlier than predicted by Svirski et al. (2012) for the $w=2$ case.

So far we have discussed the emission of X-rays from optically thick environments. However, X-rays can also be generated in optically thin environments. Following Immler et al. (2008), the X-ray emission from an optically thin region is given by

$$
L_{\mathrm{X}} \approx \int_{r}^{\infty} 4 \pi r^{2} \Lambda(T) n^{2} d r,
$$

where $\Lambda(T)$ is the effective cooling function in the $0.2-10 \mathrm{keV}$ range. Assuming optically thin thermal plasma with a temperature in the range $10^{6}-10^{8} \mathrm{~K}$ (Raymond et al. 1976), we adopt a value of $\Lambda(T) \approx 3 \times 10^{-23} \mathrm{erg} \mathrm{cm}^{3} \mathrm{~s}^{-1}$. Substituting Equation (3) into Equation (6) we get

$$
\begin{aligned}
L_{\mathrm{X}} & \approx 4 \pi \Lambda(T) \frac{K^{2}}{\left\langle\mu_{\mathrm{p}}\right\rangle^{2} m_{\mathrm{p}}^{2} r} \\
& \approx 8.6 \times 10^{41}\left(\frac{\dot{M}}{0.001 M_{\odot}}\right)^{2}\left(\frac{v_{w}}{10 \mathrm{~km} \mathrm{~s}^{-1}}\right)^{-2} .
\end{aligned}
$$

Figure 7 also shows lines of equal optically thin X-ray emission. They are plotted only to the right-hand side of the $\tau \approx 2 / 3$ line, being relevant only for optically thin regions. This suggests that in the case of a shock breakout in an optically thick CSM $\left(\tau \gtrsim c / v_{\mathrm{s}}\right)$ with a wind profile, the optically thin X-ray emission will be generated at late times roughly equal to $c / v_{\mathrm{s}}$ times the shock breakout timescale. We note that, in a wind profile, X-ray emission from optically thin material is expected to decay with the radius and therefore with time (see Figure 7).

\subsection{Radio Emission from Collisionless Shocks}

The shock going through the CSM may generate synchrotron radiation peaking at radio frequencies (e.g., Slysh 1990; Chevalier \& Fransson 1994; Chevalier 1998; Horesh et al. 2012; Krauss et al. 2012; Katz 2012). However, if the material is ionized or partially ionized then the free-free optical depth may block this radiation. In order to test if a radio signature is expected, we need to estimate the free-free optical depth (e.g., Chevalier 1981) which, for a ionized CSM with a wind profile, is given by (Lang 1999, Equation (1.223))

$$
\begin{aligned}
\tau_{\mathrm{ff}} & \approx 2.6 \times 10^{10} T_{\mathrm{e}, 4}^{-1.35} v_{10}^{-2.1} v_{\mathrm{w}, 10}^{-2} \dot{M}_{0.1}^{2} r_{15}^{-3} \\
& \propto r_{15}^{1-2 w},
\end{aligned}
$$

where $v_{10}$ is the frequency in units of $10 \mathrm{GHz}$. The free-free optical depth is so large that radio emission is not expected. However, if in some regions the CSM density profile falls significantly faster than $r^{-2}$, then the free-free absorption may be low enough for radio emission (e.g., synchrotron) to escape the CSM. We predict that if the CSM is ionized, then due to the effect of free-free absorption, the synchrotron radio emission generated in the shocked CSM may have a relatively steep radio spectrum. Therefore, it is preferable to search for this emission at high frequencies and late times after maximum light. However, the existence of radio emission is likely to be very sensitive to the exact properties of the CSM, such as density profile, symmetry, and homogeneity. We speculate that good candidates for radio emission will be $\mathrm{SNe}$ in which the wind filling factor is low, or asymmetric, or alternatively when the wind is ejected in a 
relatively short eruption, and therefore may have a steep density profile (see Section 5).

We note that Van Dyk et al. (1996) presented a search for radio emission among $10 \mathrm{SNe}$ IIn. None of the SNe in their sample was detected at radio wavelengths, but these observations were conducted 2-14 yr after the SN explosion. However, some SNe IIn were detected as bright radio sources a few years after maximum light. Examples include SN 1986J (Rupen et al. 1987; Leibundgut et al. 1991b) and SN 1978K (Ryder et al. 1993; Chugai et al. 1995).

\section{OBSERVATIONS IN CONTEXT OF THE MODEL}

As shown in Table 2 and Figures 1-5, several $\mathrm{SNe}$ in our sample show X-ray emission in the $0.2-10 \mathrm{keV}$ range. Some $\mathrm{SNe}$ are presumably detected (and maybe peaking) in X-rays near maximum optical light (e.g., SN 2011ht; SN 2010al). However, the X-ray luminosity of SN 2011 ht (see Roming et al. 2012) and SN 2010al at maximum visible light is about 0.05 and 0.3 (respectively) of their visible-light luminosity. These $\mathrm{X}$-ray luminosities are higher than predicted by Svirski et al. (2012; X-rays $10^{-4}$ of optical) for the CSM shock-breakout case. Moreover, although the X-ray light curves are consistent with a nonvariable luminosity, it is possible that they are peaking near maximum optical light. Therefore, we suggest that the optical light curves of SN 2011ht and SN 2010al, which are SNe IIn, are likely not powered by a shock breakout in CSM.

As previously reported, SN 2010jl (Chandra et al. 2012b) and SN 2006jc (Immler et al. 2007) are detected in X-rays and are peaking at late times, respectively $\gtrsim 10 t_{\text {rise }}$ and $\gtrsim 6 t_{\text {rise }}$. Moreover, the X-ray luminosity at maximum visible light is about $10^{-3}$ of the visible-light luminosity. This is roughly consistent with the predictions of Svirski et al. (2012). We discuss these SNe in detail in Sections 5.1 and 5.2.

Two other SNe in our sample, PTF 11 iqb and SN 2005db, have marginal X-ray detections. Therefore, we cannot make any firm conclusion regarding the reality and nature of this emission.

As indicated in the column $L_{X} / L_{\text {opt }}$ in Table 1 , the rest of the SNe in our sample, including all of the SLSN-I events, do not have late-time observations and/or sufficiently deep limits to evaluate their nature. Our upper limits are mostly obtained at early times $\left(\lesssim 3 t_{\text {rise }}\right)$ after the SN explosion, or at very late times $\left(\gtrsim 37 t_{\text {rise }}\right)$. We note that all of the observations of the hydrogen-poor luminous SNe were obtained at $\lesssim 2 t_{\text {rise }}$ after optical maximum light.

Recently, Chandra et al. (2012a) reported on X-ray and radio observations of another Type IIn event, SN 2006jd. This event is listed as an $\mathrm{SN}$ IIb in the IAUC SN list ${ }^{22}$ and, therefore, was not included in our sample. We note that the X-ray observations started about a year after the explosion, so there is no measurement of $L_{X} / L_{\text {opt }}$ during optical maximum light.

We conclude that deeper X-ray observations, over longer periods of time after maximum optical light, are required in order to understand the nature of SN IIn and SLSN-I events. The current null detection of hydrogen-poor luminous $\mathrm{SNe}$ in $\mathrm{X}$-rays cannot be used to reject the CSM-interaction model proposed by Quimby et al. (2011b).

\subsection{SN 2010jl (PTF 10aaxf)}

Chandra et al. (2012b) reported on the discovery of X-ray emission from SN 2010jl. Its X-ray luminosity near optical

\footnotetext{
$\overline{22}$ http://www.cbat.eps.harvard.edu/lists/Supernovae.html
}

maximum light is about $6 \times 10^{40} \mathrm{erg} \mathrm{s}^{-1}$, which is $\sim 10^{-3}$ of its $R$-band luminosity. For the case of shock breakout in CSM, Svirski et al. (2012) predicted that near optical maximum light the X-ray luminosity will be about $10^{-4}$ of the optical luminosity. However, it is possible that the bolometric optical luminosity is higher (e.g., due to metal blanketing). Moreover, we note that for $w>2$, the amount of material above the shock is smaller and the X-rays will be less effected by absorption at early times.

The Swift X-ray flux is rising with time, and is approximately consistent with a power law of the form $t^{0.4}$, where $t$ is the time since optical maximum light. Svirski et al. (2012) predicted that the hard radiation (i.e., X-ray) component of a collisionless shock will rise $\alpha t^{\beta}$, with $\beta$ between 0 and 2 when the ejecta are colliding with a wind mass that is comparable to its own mass, and with $\beta$ between 1 and $5 / 2$ when the wind is less massive than the ejecta. The fact that the X-ray emission in SN 2010j1 rises for at least 200 days suggests that its source is in an optically thick region. The reason for this is that if the $\mathrm{X}$-rays originated in the optically thin region, then we would expect to see a decline in the X-ray emission (i.e., moving along the time axis in Figure 7). We note that our observations constrain only the $0.2-10 \mathrm{keV}$ range, while the hard component can emit at energies up to $\sim 100 \mathrm{keV}$. Moreover, Svirski et al. (2012) assumed that the bound-free absorption can be neglected even at late times (i.e., the CSM is completely ionized).

Chandra et al. (2012b) reported on the analysis of the X-ray spectrum of SN 2010jl (including the proprietary data). They measured a column density at their latest epoch of $\sim 10^{24} \mathrm{~cm}^{-2}$, which is about 1000 times larger than the Galactic column density in the direction of SN 2010j1. Such a large bound-free absorption is expected if the shock velocity is below $10^{4} \mathrm{~km} \mathrm{~s}^{-1}$ (i.e., the metals are not completely ionized; Chevalier \& Irwin 2012) and the mass-loading parameter is as large as expected from a wind shock breakout (e.g., Equation (4)).

Moreover, the column density decreases by a factor of $\sim 3$ between 38 and 352 days after optical maximum light. Equation (4) predicts that between these dates, assuming $w=2$, the bound-free absorption should decrease by a factor of $\sim 9$. In order to explain the rise in column density relative to what is expected from a wind profile, we suggest that the CSM has a shell structure or that the ionization parameter changes with time. If this CSM mass density profile will be followed by a steep power-law index, we predict that the free-free absorption will be low enough and it may be possible to detect late-time radio emission from this SN (see Section 4.3). A complete analysis of all the available data, including the proprietary data, is required in order to understand the luminosity and spectral evolution of this $\mathrm{SN}$, and to give more firm predictions.

\subsection{SN 2006jc}

The X-ray light curve of SN 2006jc (Figure 2; see also Immler et al. 2008) peaked $\sim 100$ days after the explosion. The visiblelight rise time of SN 2006jc was shorter than about 15 days (Foley et al. 2007; Pastorello et al. 2008). Therefore, if this $\mathrm{SN}$ is powered by the diffusion of shock energy and if we can approximate the shock-breakout timescale as the visible-light rise time, then we can deduce that the X-rays peaked $\gtrsim 6 t_{\mathrm{br}}$.

Itagaki et al. (2006) reported that a possible eruption, with an absolute magnitude of about -13 , took place at the position of SN 2006jc about two years prior to the SN explosion (see also Pastorello et al. 2008). This pre-SN outburst of SN 2006jc may have put in place a dense shell that could provide the medium 
required for the formation of a collisionless shock. Moreover, due to the eruptive nature of the event, the outer edge of this shell may follow a density profile that falls faster than $r^{-2}$, or has a relatively sharp edge. Such a density profile is required for the X-rays to escape the shocked regions at times earlier than the 10-50 shock-breakout timescale suggested by Svirski et al. (2012).

From an analysis of the X-ray light curve of SN 2006jc, Immler et al. (2008) modeled the X-ray emission from this SN as originating in an optically thin shell at a radius of $9 \times 10^{15} \mathrm{~cm}$. In this model, the peak of the X-ray emission corresponds to the time it takes the SN ejecta, moving at $9000 \mathrm{~km} \mathrm{~s}^{-1}$, to reach the shell. The existence of a shell at this distance is consistent with the speed and time of the pre-SN outburst seen in this event.

Given the CSM velocity of $\sim 1000 \mathrm{~km} \mathrm{~s}^{-1}$ in SN 2006jc (Foley et al. 2007), we conclude that the X-ray emission in this system is roughly consistent with two explanations: (1) X-rays from an optically thin shell with a mass loss of $\sim 10^{-2} M_{\odot} \mathrm{yr}^{-1}$ (as suggested by Immler et al. 2008) or (2) X-rays from an optically thick shell with a mass loss of $\sim 1 M_{\odot} \mathrm{yr}^{-1}$. In both cases, the shell was ejected about two years prior to the $\mathrm{SN}$ explosion.

\section{CONCLUSIONS}

We present a search for X-ray emission from $28 \mathrm{SNe}$ in which it is possible that the shock breakout took place within a dense CSM. Most SNe have been observed with X-ray telescopes only around maximum optical light. SNe in our sample that have clear X-ray detections include SN 2006jc (Immler et al. 2008), SN 2010jl (Chandra et al. 2012b), SN 2011ht (Roming et al. 2012), and SN 2010al. Two additional objects, PTF 11iqb and SN 2005db, have questionable detections. The SNe in our sample that do have late-time observations were either also detected at early times or observed serendipitously at very late times. In that respect, our first conclusion is that a search for $\mathrm{X}$-ray emission both at early and late times from $\mathrm{SNe}$ is essential for constraining the properties of the CSM around their progenitors.

Our analysis suggests that some SNe IIn/Ibn, most notably SN 2010j1, have optical light curves that are likely powered by a shock breakout in CSM, while some other SNe IIn do not. However, for most of the SNe in our sample, including all of the SLSN-I events, the observations are not conclusive. Specifically, the lack of X-ray detection of SLSN-I events cannot rule out the interaction model suggested by Quimby et al. (2011b; see also Ginzburg \& Balberg 2012). We conclude that deeper observations at later times are required in order to further test this model and to estimate the fraction of SNe IIn which are powered by optically thick emission.

Given the limits found in this paper and our current understanding of these events, it will be worthwhile to monitor $\mathrm{SNe}$ IIn (as well as other classes of potentially interacting SNe; e.g., SNe IIL) with X-ray and radio instruments at timescales $\gtrsim 10$ times the rise time of the SN. In most cases presented in this paper, the $\mathrm{SN}$ rise time is in the range 10-50 days. Therefore, the recommended timescale to conduct X-ray and radio observations is between three months and two years after the SN maximum light. Given the X-ray luminosities reported in Table 1, we suggest that a luminosity sensitivity of better than $\sim 10^{41} \mathrm{erg} \mathrm{s}^{-1}$ is required in order to detect X-ray emission from these SNe.

We argue that in some cases bound-free absorption will play an important role at early and late times. Therefore, observations with the recently launched Nuclear Spectroscopic Telescope Array (NuSTAR; Harrison et al. 2010) in the 6-80 keV band may be extremely useful to test the theory and to study the physics of these collisionless shocks. Moreover, in the cases where boundfree absorption is important (e.g., $v_{\mathrm{s}} \lesssim 10^{4} \mathrm{~km} \mathrm{~s}^{-1}$; Chevalier $\&$ Irwin 2012), the spectral X-ray evolution as a function of time can be use to probe the column density above the shock at any given time, and to deduce the density profile outside the shocked regions. We also argue that in some cases, if the CSM has a steep density profile (e.g., SN 2010j1), it may be possible to detect radio emission.

Finally, we note that Katz et al. (2011) and Murase et al. (2011) predict that the collisionless shocks will generate $\mathrm{TeV}$ neutrinos. The detection of such neutrinos using IceCube (Karle et al. 2003) will be a powerful tool to test this theory and explore the physics of collisionless shocks.

We thank Ehud Nakar, Boaz Katz, and Nir Sapir for many discussions, and the anonymous referee for constructive comments. This paper is based on observations obtained with the Samuel Oschin Telescope as part of the Palomar Transient Factory project, a scientific collaboration between the California Institute of Technology, Columbia University, Las Cumbres Observatory, the Lawrence Berkeley National Laboratory, the National Energy Research Scientific Computing Center, the University of Oxford, and the Weizmann Institute of Science. E.O.O. is incumbent of the Arye Dissentshik career development chair and is grateful to support by a grant from the Israeli Ministry of Science. M.S. acknowledges support from the Royal Society. A.C. acknowledges support from LIGO, which was constructed by the California Institute of Technology and the Massachusetts Institute of Technology with funding from the National Science Foundation and operates under cooperative agreement PHY-0757058. S.R.K. and his group are partially supported by the NSF grant AST-0507734. A.G. acknowledges support by the Israeli, German-Israeli, and the U.S.-Israel Binational Science Foundations, a Minerva grant, and the Lord Sieff of Brimpton fund. The National Energy Research Scientific Computing Center, which is supported by the Office of Science of the U.S. Department of Energy under Contract No. DE-AC02-05CH11231, provided staff, computational resources, and data storage for the PTF project. P.E.N. acknowledges support from the U.S. Department of Energy Scientific Discovery through Advanced Computing program under contract DE-FG02-06ER06-04. J.S.B.'s work on PTF was supported by NSF/OIA award AST-0941742 ("RealTime Classification of Massive Time-Series Data Streams"). L.B. is supported by the NSF under grants PHY 05-51164 and AST 07-07633. M.M.K. acknowledges generous support from the Hubble Fellowship and Carnegie-Princeton Fellowship. A.V.F.'s supernova group at UC Berkeley is supported by Gary \& Cynthia Bengier, the Richard \& Rhoda Goldman Fund, the Christopher R. Redlich Fund, the TABASGO Foundation, and NSF grants AST-0908886 and AST-1211916. KAIT and its ongoing operation were made possible by donations from Sun Microsystems, Inc., the Hewlett-Packard Company, AutoScope Corporation, Lick Observatory, the NSF, the University of California, the Sylvia \& Jim Katzman Foundation, and the TABASGO Foundation.

\section{REFERENCES}

Balberg, S., \& Loeb, A. 2011, MNRAS, 414, 1715

Barbary, K., Dawson, K. S., Tokita, K., et al. 2009, ApJ, 690, 1358 
Behar, E., Dado, S., Dar, A., \& Laor, A. 2011, ApJ, 734, 26 Blanc, N., Bongard, S., Copin, Y., et al. 2005, CBET, 183, 1 Blondin, S., \& Calkins, M. 2008, CBET, 1368, 1

Blondin, S., Modjaz, M., Kirshner, R., Challis, P., \& Calkins, M. 2007, CBET, 916, 1

Boles, T., Pastorello, A., Stanishev, V., et al. 2011, CBET, 2851, 1

Challis, P., Foley, R. J., \& Berlind, P. 2010a, CBET, 2243, 1

Challis, P., Kirshner, R., \& Smith, N. 2010b, CBET, 2548, 1

Chandra, P., Ofek, E. O., Frail, D. A., et al. 2009, ATel, 2241, 1

Chandra, P., Ofek, E. O., Frail, D. A., et al. 2010, ATel, 2367, 1

Chandra, P., Chevalier, R. A., Chugai, N., et al. 2012a, ApJ, 755, 110

Chandra, P., Chevalier, R. A., Irwin, C. M., et al. 2012b, ApJL, 750, 2

Chevalier, R. A. 1981, ApJ, 251, 259

Chevalier, R. A. 1998, ApJ, 499, 810

Chevalier, R. A., \& Fransson, C. 1994, ApJ, 420, 268

Chevalier, R. A., \& Irwin, C. M. 2011, ApJL, 729, 6

Chevalier, R. A., \& Irwin, C. M. 2012, ApJL, 747, 17

Chomiuk, L., Chornock, R., Soderberg, A. M., et al. 2011, ApJ, 743, 114

Chugai, N. N., Danziger, I. J., \& Della Valle, M. 1995, MNRAS, 276, 530

Colgate, S. A. 1974, ApJ, 187, 333

Couch, S. M., Pooley, D., Wheeler, J. C., \& Milosavljević, M. 2011, ApJ, 727,104

Dickey, J. M., \& Lockman, F. J. 1990, ARA\&A, 28, 215

Dintinjana, B., Mikuz, H., Skvarc, J., et al. 2011, CBET, 2906, 1

Drake, A. J., Djorgovski, S. G., Mahabal, A., et al. 2008, CBET, 1366, 1

Drake, A. J., Djorgovski, S. G., Mahabal, A., et al. 2009a, ApJ, 696, 870

Drake, A. J., Djorgovski, S. G., Mahabal, A., et al. 2009b, CBET, 1958, 1

Drake, A. J., Djorgovski, S. G., Mahabal, A. A., et al. 2011a, ATel, 3262, 1

Drake, A. J., Djorgovski, S. G., Mahabal, A. A., et al. 2011b, ATel, 3343, 1

Drake, A. J., Djorgovski, S. G., Mahabal, A. A., et al. 2012, ATel, 3873, 1

Duszanowicz, G. 2010, CBET, 2241, 1

Falk, S. W., \& Arnett, W. D. 1977, A\&AS, 33, 515

Filippenko, A. V. 1997, ARA\&A, 35, 309

Filippenko, A. V., Li, W. D., Treffers, R. R., \& Modjaz, M. 2001, in ASP Conf. Ser. 246, Small-Telescope Astronomy on Global Scales, ed. W. P. Chen, C. Lemme, \& B. Paczyński (San Francisco, CA: ASP), 121

Filippenko, A. V., Silverman, J. M., Mostardi, R., \& Griffith, C. V. 2008, CBET, 1420,1

Foley, R. J., Smith, N., Ganeshalingam, M., et al. 2007, ApJL, 657, 105

Gal-Yam, A. 2012, Sci, 337, 927

Gehrels, N. 1986, ApJ, 303, 336

Gehrels, N., Chincarini, G., Giommi, P., et al. 2004, ApJ, 611, 1005

Gezari, S., Halpern, J. P., Grupe, D., et al. 2009, ApJ, 690, 1313

Ginzburg, S., \& Balberg, S. 2012, ApJ, 757, 178

González-Martín, O., \& Vaughan, S. 2012, A\&A, 544, A80

Grassberg, E. K., Imshennik, V. S., \& Nadyozhin, D. K. 1971, Ap\&SS, 10, 28

Hamuy, M. 2002, IAUC, 7979, 3

Harrison, F. A., Boggs, S., Christensen, F., et al. 2010, Proc. SPIE, 7732, 77320 S

Horesh, A., Kasliwal, M., Carpenter, J., et al. 2011, ATel, 3512, 1

Horesh, A., Stockdale, C., Fox, P. B., et al. 2012, ApJ, submitted (arXiv:1209.1102)

Immler, S., Modjaz, M., Landsman, W., et al. 2008, ApJL, 674, 85

Immler, S., Pooley, D., Brown, P. J., Li, W., \& Filippenko, A. V. 2007, ATel, 1284,1

Itagaki, K., Nakano, S., Puckett, T., et al. 2006, IAUC, 8762, 1

Joubert, N., \& Li, W. 2007, CBET, 912, 1

Kaiser, N., Aussel, H., Burke, B. E., et al. 2002, Proc. SPIE, 4836, 154

Karle, A., Ahrens, J., Bahcall, J. N., et al. 2003, NuPhS, 118, 388

Kasen, D., \& Bildsten, L. 2010, ApJ, 717, 245

Katz, B. 2012, MNRAS, 420, L6

Katz, B., Sapir, N., \& Waxman, E. 2011, arXiv:1106.1898

Kiewe, M., Gal-Yam, A., Arcavi, I., et al. 2012, ApJ, 744, 10

Krauss, M. I., Soderberg, A. M., Chomiuk, L., et al. 2012, ApJL, 750, 40

Lang, K. R. 1999, Astrophysical Formulae (New York: Springer)

Law, N. M., Kulkarni, S. R., Dekany, R. G., et al. 2009, PASP, 121, 1395

Leibundgut, B., Kirshner, R. P., Pinto, P. A., et al. 1991, ApJ, 372, 531

Leloudas, G., Chatzopoulos, E., Dilday, B., et al. 2012, A\&A, 541, A129

Li, W. D., Filippenko, A. V., Treffers, R. R., et al. 2000, in Cosmic Explosions, ed. S. S. Holt \& W. W. Zhang (Melville, NY: AIP), 103
Mahabal, A., Drake, A. J., Djorgovski, S. G., et al. 2011, CBET, 2941, 1 Mahabal, A. A., Drake, A. J., Djorgovski, S. G., et al. 2010, ATel, 2490, 1 Maragutti, R., Soderberg, A., Chomiuk, L., et al. 2012, ATel, 3925, 1

Matheson, T., Challis, P., Kirshner, R., \& Calkins, M. 2004, IAUC, 8268, 2 Matzner, C. D., \& McKee, C. F. 1999, ApJ, 510, 379

Maza, J., Hamuy, M., Antezana, R., et al. 2010, CBET, 2544, 1

Miller, A. A., Chornock, R., Perley, D. A., et al. 2009, ApJ, 690, 1303

Monard, L. A. G. 2005a, IAUC, 8499, 1

Monard, L. A. G. 2005b, IAUC, 8571, 1

Monard, L. A. G., \& Africa, S. 2002, IAUC, 7973, 1

Moretti, A., Campana, S., Tagliaferri, G., et al. 2004, Proc. SPIE, 5165, 232

Moriya, T. J., \& Maeda, K. 2012, ApJL, 756, 22

Moriya, T. J., \& Tominaga, N. 2012, ApJ, 747, 118

Morrison, R., \& McCammon, D. 1983, ApJ, 270, 119

Murase, K., Thompson, T. A., Lacki, B. C., \& Beacom, J. F. 2011, PhRvD, 84, 043003

Nakano, S., Itagaki, K., Puckett, T., \& Gorelli, R. 2006, CBET, 666, 1

Nakar, E., \& Sari, R. 2010, ApJ, 725, 904

Neill, J. D., Sullivan, M., Gal-Yam, A., et al. 2011, ApJ, 727, 15

Newton, J., \& Puckett, T. 2010, CBET, 2532, 1

Ofek, E. O., Cameron, P. B., Kasliwal, M. M., et al. 2007, ApJ, 659, L13

Ofek, E. O., Laher, R., Law, N., et al. 2012a, PASP, 124, 62

Ofek, E. O., Rabinak, I., Neill, J. D., et al. 2010, ApJ, 724, 1396

Ofek, E. O., Laher, R., Surace, J., et al. 2012b, PASP, 124, 854

Parisky, X., \& Li, W. 2007, CBET, 1129, 1

Parrent, J., Levitan, D., Howell, A., et al. 2011, ATel, 3510, 1

Pastorello, A., Mattila, S., Zampieri, L., et al. 2008, MNRAS, 389, 113

Pastorello, A., Smartt, S. J., Botticella, M. T., et al. 2010a, ApJL, 724, 16

Pastorello, A., Smartt, S. J., Botticella, M. T., et al. 2010b, CBET, 2413, 1

Pignata, G., Maza, J., Hamuy, M., et al. 2009, CBET, 1719, 1

Pooley, D. 2012, ATel, 4062, 1

Prieto, J. L., McMillan, R., Bakos, G., \& Grennan, D. 2011, CBET, 2903, 1

Puckett, T., Kerns, B., \& Toth, D. 2004, IAUC, 8261, 2

Quimby, R. M., Aldering, G., Wheeler, J. C., et al. 2007, ApJL, 668, 99

Quimby, R. M., Gal-Yam, A., Arcavi, I., et al. 2011a, ATel, 3841, 1

Quimby, R. M., Kulkarni, S. R., Kasliwal, M. M., et al. 2011b, Natur, 474, 487

Quimby, R. M., Kulkarni, S. R., Ofek, E., et al. 2010a, ATel, 2492, 1

Quimby, R. M., Kulkarni, S., Ofek, E., et al. 2010b, ATel, 2740, 1

Quimby, R. M., Sternberg, A., \& Matheson, T. 2011c, ATel, 3344, 1

Rabinak, I., \& Waxman, E. 2011, ApJ, 728, 63

Rakavy, G., Shaviv, G., \& Zinamon, Z. 1967, ApJ, 150, 131

Rau, A., Kulkarni, S. R., Law, N. M., et al. 2009, PASP, 121, 1334

Raymond, J. C., Cox, D. P., \& Smith, B. W. 1976, ApJ, 204, 290

Rich, D. 2010a, CBET, 2207, 1

Rich, D. 2010b, CBET, 2530, 1

Roming, P. W. A., Pritchard, T. A., Prieto, J. L., et al. 2012, ApJ, 751, 92

Rupen, M. P., van Gorkom, J. H., Knapp, G. R., Gunn, J. E., \& Schneider, D. P. 1987, AJ, 94, 61

Ryder, S., Staveley-Smith, L., Dopita, M., et al. 1993, ApJ, 416, 167

Salvo, M., Schmidt, B., Kiss, L., \& Derekas, A. 2005, IAUC, 8501, 4

Schlegel, E. M. 1990, MNRAS, 244, 269

Silverman, J. M., Filippenko, A. V., \& Foley, R. J. 2010a, CBET, 2538, 1

Silverman, J. M., Kleiser, I. K. W., Morton, A. J. L., \& Filippenko, A. V. 2010b, CBET, 2223, 1

Slysh, V. I. 1990, SvAL, 16, 339

Smartt, S. J., Weight, D., Valenti, S., et al. 2012, ATel, 3918, 1

Smith, N., Cenko, S. B., Butler, N., et al. 2012, MNRAS, 420, 1135

Smith, N., Foley, R. J., \& Filippenko, A. V. 2008, ApJ, 680, 568

Smith, N., Li, W., Foley, R. J., et al. 2007, ApJ, 666, 1116

Spergel, D. N., Bean, R., Doré, O., et al. 2007, ApJS, 170, 377

Stoll, R., Prieto, J. L., Stanek, K. Z., et al. 2011, ApJ, 730, 34

Stritzinger, M., Morrell, N., Folatelli, G., Covarrubias, R., \& Phillips, M. M. 2009, CBET, 1725, 1

Stritzinger, M., Morrell, N., \& Phillips, M. M. 2010, CBET, 2220, 1

Svirski, G., Nakar, E., \& Sari, R. 2012, ApJ, 759, 108

Van Dyk, S. D., Weiler, K. W., Sramek, R. A., et al. 1996, AJ, 111, 1271

Weaver, T. A. 1976, ApJS, 32, 233

Woosley, S. E., Blinnikov, S., \& Heger, A. 2007, Natur, 450, 390

Yaron, O., \& Gal-Yam, A. 2012, PASP, 124, 668 